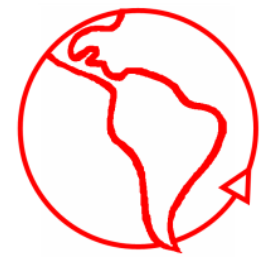

\title{
Niklas Luhmann: "Sociología de la religión" [2009, México D.F.: Herder/UIA, 288 pp.]
}

Niklas Luhmann: "Sociología de la religión" [2009, México D.F.: Herder/UIA, 288 pp.]

Marco Ornelas

Departamento de ciencias sociales y políticas, Universidad Iberoamericana, México

\section{EL CONTENIDO DEL LIBRO}

El libro es un compendio de las dos investigaciones teórico-sociológicas más largas y exhaustivas sobre religión hechas por Niklas Luhmann (1927-1998) hasta antes de la publicación póstuma de su monografía temática La religión de la sociedad, publicada en alemán en el año 2000 y de la que ya existe traducción española (Luhmann 2007b). Se trata de La dogmática religiosa y la evolución de las sociedades (Luhmann 1977) y de El proceso de diferenciación de la religión (Luhmann 1989). Los dos trabajos presentan un aparato crítico impresionante -juntos suman cerca de 400 notas al pie-, haciendo los honores a las legendarias fichas bibliográficas de trabajo de este notable sociólogo alemán de finales del siglo xx.

El valor de estos dos artículos, a más de diez años de distancia entre sí, estriba en que muestran de manera expresa la labor de "picapedrero" realizada por Luhmann y que le permitió no sólo delinear los rasgos esenciales del sistema religioso de la sociedad, sino también fincar sólidamente las características centrales de su teoría general de los sistemas sociales, de su teoría de la sociedad moderna. En los doce años que separan la publicación de los dos textos apareció la obra que marcó el vuelco definitivo de la teoría a los sistemas sociales autorreferentes o autopoiéticos en su operación: Sistemas sociales. Lineamientos para una teoría general, publicada en Alemania en 1984 y de la que también se cuenta con traducción española (Luhmann 1991). Con esto se confirma lo que Roger O'Toole llama el primer artículo del credo de la subdisciplina (de la sociología religiosa): "...el entendimiento sociológico de la religión es un elemento indispensable para el análisis teórico de la sociedad y la cultura" (O’Toole 1985: 2). ${ }^{1}$

\footnotetext{
1 "...the sociological understanding of religion is an indispensable element in the theoretical analysis of society and culture" (las traducciones son del autor de la reseña).
}

\section{REVISTA MAD - UNIVERSIDAD DE CHILE}

Revista del Magíster en Análisis Sistémico Aplicado a la Sociedad

Facultad de Ciencias Sociales, Departamento de Antropología.

Universidad de Chile

www.revistamad.uchile.cl 
La dogmática religiosa y la evolución de las sociedades es solidaria con la tradición de pensamiento social que aborda el estudio de las religiones desde una perspectiva funcionalista, evolucionista y científica. Sin pretender ser exhaustivo en el recuento, podrían ubicarse en ella a Edward Tylor (Cultura primitiva, 1871), a James George Frazer (La rama dorada, 1890), a Emile Durkheim (Las formas elementales de la vida religiosa, 1912), a Bronislaw Malinowsky (Los argonautas del Pacífico Occidental, 1922) y a Edward Evans-Pritchard (Brujería, magia y oráculos entre los Azande, 1937). En general, el análisis funcionalista de la religión pone su atención en los mutuos apoyos y determinaciones entre las prácticas y creencias religiosas y el entramado todo de la vida social. Las religiones, a la vez que explican características centrales de la vida social (parentesco, actividades productivas, control político), quedan explicadas por ellas.

Los rituales y las creencias religiosas son los dos componentes esenciales de toda religión. La agudeza del análisis luhmanniano consiste en poner estos dos elementos en interconexión y constante tensión, de manera que los rituales y las doctrinas religiosas son analizados dinámicamente en un proceso de evolución socio-histórica. En tanto que el ritual se funda y supone siempre una interacción entre presentes, reporta un excedente de socialidad -aunque no solamente: también propicia éxtasis místicos. ${ }^{2}$ El ritual socializa, esto es, proporciona de manera inmediata un sentido religioso compartido. En cambio, las creencias o doctrinas religiosas siempre adoptan una significación opuesta: suponen un sentido religioso que se abstrae de situaciones interactivas inmediatas. También suponen la aparición de sociedades más complejas con escritura y medios técnicos capaces de fijar significados religiosos variados. Es así que la creencia o doctrina sancionada socialmente "desocializa" el mundo. Luhmann lo pone en estos términos:

(...) la gradual desocialización del mundo es el resultado de procesos evolutivos complicados... En términos de simbolismo, la desocialización del mundo es en mucho idéntica al desarrollo de un simbolismo religioso abstracto. Tal simbolismo ya no es dependiente incondicionalmente de una confirmación directa en la interacción social (Luhmann 2009: 100).

A reserva de invitar a la lectura del texto y al examen detallado del argumento, podría decirse que mientras los dogmas de fe parecen más atrevi-

${ }^{2}$ Compárese por ejemplo a James (2002). 
dos y sacados de la chistera, mayor es la necesidad de la religión de compatibilizarse con procesos societales altamente complejos y de afirmarse como garante de membresía, esto es, como organización religiosa frente a credos alternativos.

Por otra parte, El proceso de diferenciación de la religión contiene un esfuerzo por explicar las transformaciones experimentadas en la religión y que a partir del siglo xv desencadenaron el surgimiento de la sociedad moderna. La sociedad moderna supone la co-evolución de sistemas sociales en el camino de la especialización y autonomización comunicativas. Si en sociedades premodernas o de antiguo régimen los distintos ámbitos de actividad encontraban un aliado seguro en las instituciones religiosas -la redundancia social o reverberación religiosa aseguraba el pago puntual de la renta de la tierra, la obediencia al rey, el curso debido de una pasión amorosa-, Luhmann tratará de explicar cómo es que esta situación llegó a su fin. En sus palabras:

El proceso de diferenciación envuelve una renuncia a la redundancia. La religión no asegura hoy ni contra la inflación ni contra un indeseado cambio de gobierno, ni contra el desenlace de una pasión, ni contra la refutación científica de las propias teorías. No puede inmiscuirse en otros sistemas de funciones (Luhmann 2009: 195).

2. EL ESTUDiO DE LA RELIGIÓN DENTRO DE UNA TEORÍA DE LA SOCIEDAD MODERNA

Como se puede apreciar, la dificultad de leer a Luhmann está en que no elaboró una teoría más de la religión (o para el caso: de la política, del derecho, de la economía, de la educación, de la ciencia), sino en el hecho de que su teoría de la religión está inserta en una teoría de la sociedad moderna (Luhmann 2007a). Además, esta teoría puede presentarse con todo derecho como nuevo paradigma de la ciencia social, al menos por desechar expresamente los antiguos presupuestos de toda teoría social hasta el día de hoy, y que considera nuevos obstáculos epistemológicos para quienes hacen sociología. Ellos son cuatro:

(1) Que la sociedad está constituida por hombres concretos y por relaciones entre seres humanos.

(2) Que, por consiguiente, la sociedad se establece -o por lo menos se integraa través del consenso de los seres humanos, de la concordancia de sus opiniones y de la complementariedad de sus objetivos. 
(3) Que las sociedades son unidades regionales, territorialmente delimitadas, por lo cual Brasil es una sociedad distinta de Tailandia; los Estados Unidos son una sociedad distinta de lo que hasta hace poco se llamó Unión Soviética, y también Uruguay es una sociedad distinta de Paraguay.

(4) Y que, por tanto, las sociedades pueden observarse desde el exterior como grupos de seres humanos o como territorios (Luhmann 2007a: 11-12).

Algunos sociólogos aún hoy día consideran estas afirmaciones como temerarias. Pero lo cierto es que están contenidas en las primeras páginas de la teoría de la sociedad, mientras que Luhmann dedicó las restantes 900 del libro, así como 30 años de su vida profesional como sociólogo, a tratar de explicar cómo esto era posible. En 1984 Niklas Luhmann fue invitado por la American Sociological Association a San Antonio, Texas, a encabezar un simposio sobre su teoría de sistemas sociales. Roger O'Toole, quien coordinara los esfuerzos por publicar los debates, se refirió entonces a la teoría social de Luhmann en esta forma:

La empresa intelectual en curso de Luhmann es ambiciosa, abstracta, compleja y en extremo provocativa. En deuda con Parsons y Husserl entre otros, es un intento radicalmente original de formular una teoría de los sistemas sociales de aplicación universal. Como una versión de la teoría general de sistemas que pretende remplazar o "englobar" teorías que le preceden, la teoría de Luhmann reivindica una relevancia única para el mundo moderno... En su representación de la "tradición humanística" de la teoría social, que comprende por igual el liberalismo, el marxismo, la teoría crítica y la fenomenología entre otras perspectivas, la teoría de Luhmann apunta a una contra-revolución epistemológica. Postula, ni más ni menos, la abolición del sujeto humano como punto central del pensamiento social. Al considerar a los sistemas sociales compuestos de unidades de comunicación más que de seres humanos, concibe a los individuos tan sólo como entorno de los sistemas sociales. Según Luhmann, sin sistema no puede haber sentido, ni experiencia ni acción. Así, no son los sujetos humanos los que determinan el sentido, sino más bien al contrario, son los sistemas de sentido producidos históricamente los que determinan a los sujetos como seres significantes (O'Toole 1985: 1).

\section{LA RELIGIÓN COMO FORMA DE SENTIDO, Y EL SENTIDO COMO FORMA DE TRA- TO DE LA SOCIEDAD CON LA COMPLEJIDAD}

La sociología religiosa luhmanniana rechaza los dos posibles usos sociales atribuidos por la sociología clásica a la religión: hacerla responsable de la cohesión social (Durkheim) o considerarla un poderoso motivo para la ac- 
ción (Weber). Luhmann abre su participación en el simposio de San Antonio, Texas, referido más arriba, con la siguiente frase:

La teoría sociológica en su actual fase Alejandrina parece preocupada con la interpretación de sus autores clásicos. Hacer sociología de la religión significa hacer investigación empírica sobre personas o instituciones presumiblemente religiosas; significa regresar a Emile Durkheim o a Max Weber en busca de inspiración teórica. Entonces se supone que la religión es un factor integrativo al nivel de las sociedades totales y también un factor motivacional al nivel de los individuos. En ambos niveles proporciona un sentido del sentido, una "realidad última" significante. Todos los símbolos y valores que operan en este nivel más alto de últimos recursos pueden calificar como religión -trátese de una religión civil en el sentido de Rousseau o de Bellah (Luhmann 1985: 5).

Para Luhmann, la religión cumple la función social de restringir el sentido de lo que acontece en el mundo con ideas religiosas. La religión es el mecanismo que por milenios realizó la tarea que deben salvar las comunicaciones modernas -con otros dispositivos: los medios simbólicos- para hacerse efectivas, a saber: dar por sentado un sentido determinable. Ante la indeterminación de sentido, ante los múltiples significados que pueden tomar los acontecimientos del mundo, la religión afirma un sentido determinable con ideas religiosas. Como se vio atrás, esto se hace en sociedades simples mediante prácticas rituales, como ritualización de toda interacción social (la religión y la sociedad son una y la misma cosa). En la medida en que las sociedades se hacen más complejas, en la medida en que aparecen la escritura y medios técnicos para la fijación de sentidos posibles de las cosas, aparece la doctrina institucionalmente sancionada por iglesias.

Así, surgen las condiciones sociales que hacen posible los arcaísmos (semánticas que no se corresponden con la estructura social vigente o que suponen una estructura social ya rebasada) o, como lo pusiera Koselleck (1993: 333ss.), que remiten a la contemporaneidad de lo anacrónico o al anacronismo de lo contemporáneo (un buen ejemplo de esto: la misa católica). Pero en el origen, la función social de la religión es el problema de toda comunicación: el problema de su aceptación con un sentido (pre)determinable. La religión como sistema de comunicación reduce la complejidad del mundo al tornar determinable lo indeterminable. De nuevo Luhmann en San Antonio, Texas:

Los sistemas de comunicación desarrollan una forma especial de trato con la complejidad, esto es, introducen una representación de la complejidad del 
mundo en el sistema. Llamo a esta representación de la complejidad "sentido" -eludiendo todas las connotaciones subjetivas, psicológicas o trascendentales del término. La función del sentido es dar entrada a todos los temas posibles de la comunicación. El sentido coloca todos los tópicos concretos en un horizonte de posibilidades ulteriores y finalmente en el mundo de todas las posibilidades (Luhmann 1985: 7).

No deberá extrañar que, en su última y definitiva formulación teórica, la religión sea considerada como forma de sentido (Luhmann 2007b: 9ss).

4. LOS SISTEMAS SOCIALES AUTORREFERENTES O AUTOPOIÉTICOS SON SISTEMAS QUE OBSERVAN

La diferenciación de la religión es el mismo proceso que, desde una perspectiva no religiosa, tuvieron que enfrentar otros sistemas sociales en forma paralela: la economía, la política, el arte, el derecho, la ciencia. Así, tenemos no sólo una sociedad mundial compuesta de sistemas de comunicación, sino de sistemas sociales que observan. Los sistemas de comunicación son capaces de distinguir entre sí mismos (autorreferencia) y cosas distintas de sí (heterorreferencia), que no es otra cosa sino la consecuencia de la circularidad recursiva de todo sentido, de toda religión y de toda comunicación en la sociedad moderna. Las bases de esta revolución epistemológica tienen un triple referente: la neurobiología (El árbol del conocimiento: las bases biológicas del entendimiento humano de Humberto Maturana y Francisco Varela, 1984), la matemática (Laws of Form de George Spencer-Brown, 1969) y la cibernética de segundo orden (Observing Systems de Heinz von Foerster, 1981). ${ }^{3}$

Si los sistemas de comunicación del mundo actual admiten observaciones de sí (autorreferencias) así como observaciones cruzadas (heterorreferencias), entonces es posible distinguir a la dogmática religiosa y jurídica, y a la teoría política, económica y del conocimiento como autorreferencias de los sistemas sociales correspondientes (religión, derecho, política, economía, ciencia), y a las acusaciones de las distintas iglesias contra la inmundicia de los negocios, la política y las leyes como una heterorreferencia del sistema religioso (una observación cruzada proveniente de la religión). Piénsese, como ejemplo vigente, en la reciente polémica de la iglesia católica mexicana contra la ley que despenaliza el aborto en la Ciudad de México. Aunque también la distinción auto/heterorreferencia pu-

${ }^{3}$ Compárese a Luhmann (1996, 55ss) y a Torres Nafarrate (2011). 
diera ayudar a desanudar "problemas" de la subdisciplina que surgen a falta de una teoría adecuada para analizarlos, como el relativo a la dificultad que experimentan los sociólogos de la religión creyentes para estudiar científicamente... ¡ a las religiones! (Reed 1974).RM

\section{REFERENCIAS}

James, W. (2002). Las variedades de la experiencia religiosa: Estudio de la naturaleza humana. Barcelona: Península.

Koselleck, R. (1993). Futuro pasado. Para una semántica de los tiempos históricos. Barcelona: Paidós.

Luhmann, N. (1977). Religiöse Dogmatik und Gesellschaftliche Evolution. En Funktion der Religion (pp. 77-181). Frankfurt a.M.: Suhrkamp.

Luhmann, N. (1985). Society, Meaning, Religion: Based on Self-Reference. Sociological Analysis, 46(1), 5-20.

Luhmann, N. (1989). Die Ausdifferenzierung der Religion. En Gesellschaftsstruktur und Semantik. Studien zur Wissenssoziologie der modernen Gesellschaft, Vol. 3 (pp. 259-357). Frankfurt a.M.: Suhrkamp.

Luhmann, N. (1991). Sistemas sociales. Lineamientos para una teoría general. México D.F.: Alianza/UIA.

Luhmann, N. (1996). La ciencia de la sociedad. México D.F.: Anthropos/ITESO/UIA.

Luhmann, N. (2007a). La sociedad de la sociedad. México D.F.: Herder/UIA.

Luhmann, N. (2007b). La religión de la sociedad. Madrid: Trotta.

Luhmann, N. (2009). Sociología de la religión. México D.F.: Herder/UIA.

O'Toole, R. (1985). Religion and Ultimate Paradox: A Symposium on Aspects of the Sociology of Niklas Luhmann: Introduction. Sociological Analysis, 46(1), 1-3.

Reed, M. S. (1974). The Sociology of the Sociology of Religion: A Report on Research in Progress. Review of Religious Research, 15(3), 157-167.

Torres Nafarrate, J. (2011). El sentido como "la diferencia específica" del concepto de observador en Luhmann. En J. Torres Nafarrate \& D. Rodríguez (Eds.), La sociedad como pasión. Aportes a la teoría de la sociedad de Niklas Luhmann (pp. 299-334). México D.F.: UIA.

SOBRE EL AUTOR

Marco Ornelas es Doctor en Ciencias Sociales por El Colegio de Sonora, México. Becario Posdoctoral adscrito al Departamento de Ciencias Sociales y Políticas de la Universidad Iberoamericana, México.

CONTACTO: marcornelas@hotmail.com

Recibido: Noviembre 2014

Aceptado: Enero 2015 